\title{
Una salida al esencialismo. Lógicas procedimentales y posibilidades para la intervención social
}

A way out of essentialism. Procedural logics and possibilities for social intervention

Ángel Marroquin Pinto

Magister en Trabajo Social, Pontificia Universidad Católica de Chile. Profesor instructor adjunto Escuela de Trabajo SocialUC Encargado Area de Metodología e Innovación, Fundación Proyecto Propio. aamarroq@uc.cl Dirección Postal: Las Hortencias 2882, providencia, Santiago de Chile.

\section{Resumen}

En los usos del concepto de intervención social parece existir una tensión lógica entre lo que Adorno llama pensamiento identitario y nuevas formas de encarar la complejidad, como puede ser la tecnologia social. Dentro de este último enfoque se despliega una reflexión acerca de la procedimentalidad y se sostiene que esta responde a una herramienta conceptual capaz de producir intervenciones bajo propiedades y estándares que, al complejizar la intervención, aumentan con ello la eficacia y eficiencia de la gestión.

Palabras claves. Intervención social, pensamiento identitario, procedimentalidad.

\section{Abstract}

It seems to be a tension related to the use of the concept of social intervention between what Adorno calls identitarian thinking and new ways of facing complexity, such as social technology. A reflection about proceduralism unfolds within this latter approach, and argues that it refers to a conceptual tool capable of producing interventions under standards whic complexity as well as the effectiveness and efficacy of management.

Key words. Social intervention, identitarian thinking, procedurality.

\section{Introducción}

Conocida es la anécdota que nos muestra a Galileo retractándose de sus postulados científicos ante la inquisición (bajo amenaza de ser quemado en la hoguera por su descubrimiento de que la tierra giraba alrededor del sol y no al revés como aseveraba la Iglesia) mientras murmura: "y sin embargo se mueve". Igualmente parece suceder con la intervención social. A pesar de los interventores y de las organizaciones que proveen estos servicios, los efectos de sus afanes parecen dar buenos resultados. La complejidad parece ser reducida por los propios clientes que, a fin de cuentas, parecen notar que no es tan grave contar con sus propios recursos para resolver sus demandas, en comparación al tiempo que pierden deambulando por oficinas o esperando el cumplimiento de promesas.

Tras esta caricatura anida un análisis fuertemente arraigado en las instituciones y organizaciones que proveen intervenciones: el que los portadores de demandas o problemas son las personas y que las encargadas de encontrar soluciones a estos son las intervenciones que se desprenden de las políticas que orientan a estas entidades.

Lo que esta clase de análisis releva es una forma de ver que rodea y permite explicar los fenómenos que ocurren dentro de la provisión de servicios (llamada, en este caso intervención social), asociada a la cultura institucional y/o la formación académica de los interventores. ¿Es así? Esta forma de hacer intervención estaría compuesta por la complicidad de formas de ver que impiden iluminar lo que no es visible a primera vista: lógicas de intervención esencialistas que conviven con tecnologías sociales aplicadas. Lo más anacrónico al lado de lo más moderno. He aquí la paradoja.

En lo que sigue nos proponemos iluminar esta paradoja a la vez que pensar condiciones de posibilidad 
que permitan desinstalar lógicas esencialistas y ac tualizar las tecnologías sociales.

Bajo ningún punto de vista se subentiende que lo tecnológico sea lo bueno y lo esencialista algo malo. Suponemos cuponos que se despliega la intervención hoy requiere herramientas que logren reducir complejidad con calidad. Así como a nadie, enfermo o sano, se le ocurriría pensar que, actualmente, es mejor usar un bisturí a una laparoscopía en una operación quirúrgica donde está en juego la vida del paciente, tampoco en la intervención social es posible pensar que los enfoques inspirados por la base positivista a ongen del trabajo social pueden actualmente dar cuenta de los complejos fenómenos sociales que se afrontan por los interventores en su quehacer diario.

Antes de continuar vale hacer una precisión. Nuestro campo de estudio son las lógicas de conocimiento que dan origen a las prácticas conocidas como intervenciones sociales (como lo ha planteado Teresa Matus (Matus. 2002). Esto implica que ev mente en la ejecución de estas prácticas preexisten formas de ver lo social y, en cierta medida, un cálculo previo de sus posibilidades y limitaciones lógicas. Por otra parte, implica también hacerse cargo de que quien pretenda hablar de intervención social, debe señalar explícitamente sus filiaciones, y desde llas, plantear divergencias frente a otros enfoques. Hace bastante tiempo que el monoteísmo en trabajo social es solo una forma de mala fe.

Lógica postmetafísica y racionalidad procedimental En su texto Pensamiento Postmetafísico (Habermas, 1990:16), el autor revisa los fundamentos del pensamiento actual en el horizonte histórico de la modernidad. Señala que existirían una serie de movimientos que signarían el desarrollo del pensamiento moderno en el que destacan, como "figura del espíritu", la filosofía analítica, la fenomenología el marxismo occidental y el estructuralismo.

El autor menciona lo que denomina como cuatro motivos del pensamiento moderno que, en su conjunto, brindarían una consistencia propiamente moderna a cada una de estas escuelas filosóficas: 1) pensamiento postmetafísico, 2) giro lingüístico, 3 ) carácter situado de la razón y 4) inversión del primado de la teoría sobre la praxis

Respecto al primero de estos motivos, señala $\mathrm{Ha}$ bermas:

Solo bajo las premisas de un pensamiento postme- tafísico, que hace tranquila profesión de su carácter de tal, se desmorona ese concepto enfático de teoría, que pretendía hacer inteligible no solamente el mundo de los hombres, sino también las propias estructuras internas de la naturaleza. (Habermas, 1990:16-18).

La consecuencia más gravitante que resulta de la aparición de este pensamiento es que viene a romper con el concepto de la teoría: "que caracterizó a la tradición, a la idea de un pensamiento capaz de abarcar la totalidad, a la pretensión de un acceso privilegiado a la verdad" (Habermas, 1990:17), y thecho de que responde a la emergencia de un mergencia de un tipo de racionalidad capaz de cimentar y sostener el pensamiento científico moderno. Señala Habermas, -y he aquí el punto al que quiero llegar-, que: "En adelante sería la racionalidad procedimental que caracteriza al método científico la encargada de decidir si una oración puede en principio ser verdadera o falsa" (p.17).

Es entonces, en y a través de la modernidad, su horizonte histórico, que se produce la emergencia y aparición de un tipo de racionalidad anclada en la ciencia y expresada a través de una secuencia lógica adscrita al método científico ${ }^{1}$. Por lo anterior, es que cobra sentido el que la modernidad se caracterice por el despliegue de la racionalidad procedimental en que, tal como señala Habermas (1990):

\section{Las modernas ciencias experimentales y una moral} que se ha vuelto autónoma y solo se fian ya de la racionalidad de sus propios avances y de su procedimiento, a saber: del método del conocimiento cientifico o del punto de vista abstracto desde el que es posible resolver algo en moral (...) esta depende de la racionalidad de los procedimientos conforme a los que se tratan de resolver los problemas -empiricos y teóricos- en la comunidad de investigadores y en la esfera de la ciencia organizada, y problemas práctico-morales en la comunidad de los ciudadanos de un Fstado democrático y en el sistema jurídico. (Pp. 17-18).

La consecuencia de este despliegue lógico-procedimental se encuentra en el hecho de que el individuo pierde una referencia a cualquier tipo de totalidad ontológica pero, especialmente, en el que la racionalidad procedimental se desarrolla y amplía, con la modernidad, en los campos de las leyes, la política y la ciencia simultáneamente. Este despliegue no quiere decir que las formas previas, ontologizantes, se hayan disipado.
Esencialismo y pensamiento identitario

El hecho de que el pensamiento postmetafísico se re decir que los procesos de modernización hagan suyos sus principios. Al respecto observa Lechner (2007): "La modernización conlleva un acelerado proceso de diferenciación que incrementa el dinamismo de la sociedad, pero que también agudiza los fenómenos de desintegración y fragmentación. Las dos caras del proceso generan incertidumbre y un sentimiento de desamparo" (p. 365). Así como puede coexistir moder es posible paradojizar la relación entre la pervivencia de lógicas binarias y tecnología social en la intervención social

\section{Vamos con la primera de ellas.}

Cuando hacemos referencia a formas tradicionales de hacer nos referimos a aquellas maneras de pensar la intervención que encuentran su raíz en lo que Adorno llamó pensamiento identitario, es decir: "el modo de pensar esencialista, substancialista, el que lleva a tratar las actividades o preferencias propias de ciertos individuos o de ciertos grupos de una cierta sociedad, en un determinado momento, como propiedades sustanciales, inscritas de una vez y para siempre en una especie de esencia biológica o, lo que no es mejor, de una esencia cultural, que lleva a los mismos errores en la comcon sociedades diferentes pero sí entre períodos sucesivos de una misma sociedad" (Adorno y Horkheimer, 1987). De esta forma, el referido esencialismo se vuelve ese acuerdo tácito, prereflexivo, inmediato, sobre el sentido del mundo que es el fundamento y filtro de las experiencias presentes y que configura el carácter supuestamente técnico que adopta una intervención social y que paradojalmente cambia sin cambiar el foco desde el que provee su oferta.

Solo a modo de ilustración. Es habitual escuchar a algunos operadores sociales señalar cosas como "los amigos influyen en el consumo de drogas de los adolescentes", "la familia es fundamental para la rehabilitación", etc. Estas frases muestran que partir de categorís a priori, incuestionadas, es elcamino más fácil para arribar a lo que Adorno llama pensamiento indentitario $\mathrm{y}$, por lo tanto, a prácticas esencialistas uniformes, totales.

Por otra parte, el pensamiento identitario, en su núcleo, se refiere a la equiparación de ser y pensamiento, en palabras de Habermas (1990) "destacando la supremacía del Uno sobre lo múltiple" (p.25) a la vez que conlleva implícitamente una lógica de la repetición. En ese sentido es que Adorno (1987) asocia la idea de pensamiento identitario a la de mito: "se recupera siempre lo mismo, es decir, repite un mismo principio explicativo de lo real" (p. 12).

Así, delante de una realidad compleja se usa el esencialismo como una forma de explicar de ordenar, de dar sentido al mundo. Lo significativo de esto es observar determinadas sociedades o períodos históricos donde el esencialismo sobredetermina de tal modo lo real que lo hegemoniza y brota así encarnado como un discurso sobre lo real.

En síntesis, es posible sostener que el esencialismo presenta como características ${ }^{2}$

1. Una totalización del Uno, rompiendo de esta forma la tensión con lo múltiple.

2. Una lógica preargumentativa que demanda la adhesión de los sujetos.

3. Un cierto principio metafísico que se presenta como ontología en cuanto existe una especie de naturalización del ser

4. Una eximición del trabajo del concepto, en cuanto existe una aceptación esencialista de algo como dado.

Al presentarse inserto en la propia estructura de lo real, el pensamiento identitario se inscribe en los hechos (Adorno, pp. 19). Esto es relevante al pensar en la intervención social, al modo que Adorn y Horkheimer se refirieron al Iluminismo como promesa de progreso o vía regia de desmitificación que ha llevado a lo que los autores llaman, estado (Adorno y Horkheimer, 987). Ese esencialismo preformativo determina incluso el propio concepto de "claridad" en el que los discursos de interventor e intervenido deben adecuarse (campo semántico de la intervención).

A modo de ejemplo, en este punto podemos recurrir a las variadas experiencias de simulación mutua entre los operadores sociales y sus públicos, o lo que Lidia Girola ha llamado el hacer "como sí" (Araujo, 2009).

Bajo ningún punto de vista el pensamiento identitario resulta hegemónico en la intervención, aún 
cuando puede parecérnoslo al leer y escuchar la manera en que las instituciones se refieren a sus publicos y estos señalan sus expectativas respecto a los primeros. Más bien parece suceder que esta form lógica constituye una forma de ver que, como dijimos anteriormente, pervive en relacion de hibridaje con formas lógicas distintas en la intervención.

\section{Tecnología social y procedimentalidad}

Antes de dilucidar la lógica subyacente a lo que denominaremos tecnología social, vale hacer una breve precisión respecto al concepto desde el que proviene, la tekné.

Con la palabra tekné los griegos nombraban el saber y hacer que llevaban adelante los artesanos y también el arte más elevado y las bellas artes. Según señala Jorge Acevedo, hasta Platón, las palabras tekné y epistéme se: "presentan unidas; ambas designan el conocer en el más amplio sentido. Esto hizo que fuese habitual pensar en Prometeo como un filósofo" (Acevedo, 1990: 68). Recordemos de pasada aquí a los presocráticos como Tales, Anaximadro o Anaxágoras, que hicieron de la solución de problemas concretos como la reorientación de un río, la auscultación de los astros e incluso la meteorología parte de su trabajo en tanto filósofos.

Aun cuando tekné y episteme aparezcan unidas, lo decisivo de la tekné, agrega Acevedo (1990) siguiendo a Heidegger, "no estriba en hacer y manipular tampoco, en aplicar medios; lo decisivo en ella consiste en ser el modo de des-ocultar, de aquello que no se pro-duce por sí mismo" (p.69). He aquí que la técnica sea una forma de verdad, sin embargo, imitada, como Es posible observar que arte y tekné comparten una misma raíz, vinculada, en este caso a la trasformación del objeto y a una cierta verdad sobre él. Este uso proviene de la revolución industrial, que corresponde a aquel momento en que la técnica adquiere esta vinculación hegemónica con los instrumentos. Y es que como señah Carvajal (2010), o que hace diferente la nocion griega de tekné de la moderna técnica es que el primero: "consiste en que toda técnica se organiza como campo de procedimiento en función de unos principios no técnicos sino discursivos" ( $\mathrm{s} / \mathrm{p})$. Lo que entendemos, por lo tanto, como tecnología (derivado de técnica) posee raíces conceptuales que no responden únicamente de la trasformación del objeto o la creación de instrumentos.

He aquí la principal limitación de la técnica y su peligro:
Los limites de la postura técnica llaman la atención sobre el carácter excluyente de esa actitud, que le impide ver esos, sus propios limites, y que obstaculiza todo intento de cooperar en el advenimiento de un nuevo destino, en que se acoja lo técnico sin dejarse avasallar por el imperar de su esencia. (Acevedo, 1990:69).

Bajo ningún punto de vista la técnica se encuentra más allá de la ética, aún cuando sea una forma de verdad. La palabra tecnología refiere compilación, al establecimiento de una recolección de saber, a un campo de aplicación de cierta técnica.

Actualmente los avances en tecnologías de la comunicación, de la información y de medios no se plantean si es necesaria más o menos tecnología, sino la inevitabilidad de su inclusión en la administración pública y privada como una perspectiva filosófica capaz de producir procedimientos que hagan la organización social posible (Thygersen, 2011). En trabajo social la práctica basada en la evidencia ha avanzado en el reconocimiento de la tecnología como un medio para dar al trabajo social el estatus de profesión (Hall, 2008).

¿Puede un concepto como este dar cuenta de las relaciones en que los seres humanos desarrollamos nuestra vida colectiva?

El concepto aparece claramente cuando pensamos en nuestra necesidad de organizarnos para llevar adelante nuestra vida en común. En este proceso, como todos nosotros sabemos por experiencia propia, cumplimos diversas funciones y roles, - ser padres, madres, hijos, trabajadores, alumnos, etc.-que implican vinculación con el mercado, el Estado y las propias comunidades donde vivimos. Evidentemente esto no es sencillo pues implica también la aparición de conflictos, la necesidad de llegar a acuerdos y la aparición de dificultades nuevas. A la aparición creciente de estas dificultades las denominamos complejidad (y es que cuando aumentan las alternativas posibles de ser elegidas, se hace más difícil elegir, como sabemos cuando vemos la repisa de un supermercado o tenemos que elegir con qué vestirnos)

La pregunta clave en este sentido parece ser: ¿Cómo hacer frente a estas dificultades?, y sobre todo, ¿Cómo resolverlas?

Es aquí que el concepto de tecnología, (es decir, un conjunto de reflexiones acerca de las normas sobre un saber específico), nos resulta útil. Por lo tanto, cuando hablamos de tecnología social decimos que la reflexión que nos proponemos se despliega acerca de cómo producir coordinación al servicio de las relaciones que los seres humanos desarrollamos cotidianamente. En otras palabras, cómo hacemos para lograr trabajar juntos y qué clase de mecanismos pueden contribuir a organizar el aporte que cada miembro hace para logros comunes. ¿Para que queremos lograr esto?. Para promover relaciones de entre los diversos actores con los que nos relacionamos diariamente; el Estado (a través de sus políticas públicas, el municipio, etc.); nuestro propio barrio y el mundo de la empresa (empresas aledañas $\mathrm{o}$ a las que accedemos en busca de servicios). Esto es promover el relacionamiento, esto es sumar.

Finalmente, cuando hablamos de tecnología social, nos referimos, extrapolando lo anterior, a la reflexión organizada acerca del campo de la intervención social, es decir, a la reflexión producida sobre la técnica, el hacer, de la intervención. Esta reflexión, sostenemos, produce principios-formas, uno de ellos es el que denominamos como procedimentalidad. Este principio (Figura $\mathrm{N}^{\circ} 1$ ):

- Permite explicar cómo se produce coordinación por medio del uso de diversos mecanismos.

- Permite explicar que los procedimientos nacen del consenso entre los actores.

- Permite explicar la intervención social por medio de estrategias de orientación.

En este sentido es que hablamos de sistemas de coordinación, que permiten producir y gestionar intervenciones sociales en contextos complejos y que, a través de ellas, buscan promover autonomía en los sujetos (plan ético) a través de la trasferencia de competencias, habilidades y procedimientos.

FIGURA No1

TECNOLOGÍA SOCIAL Y PROCEDIMIENTALIDAD

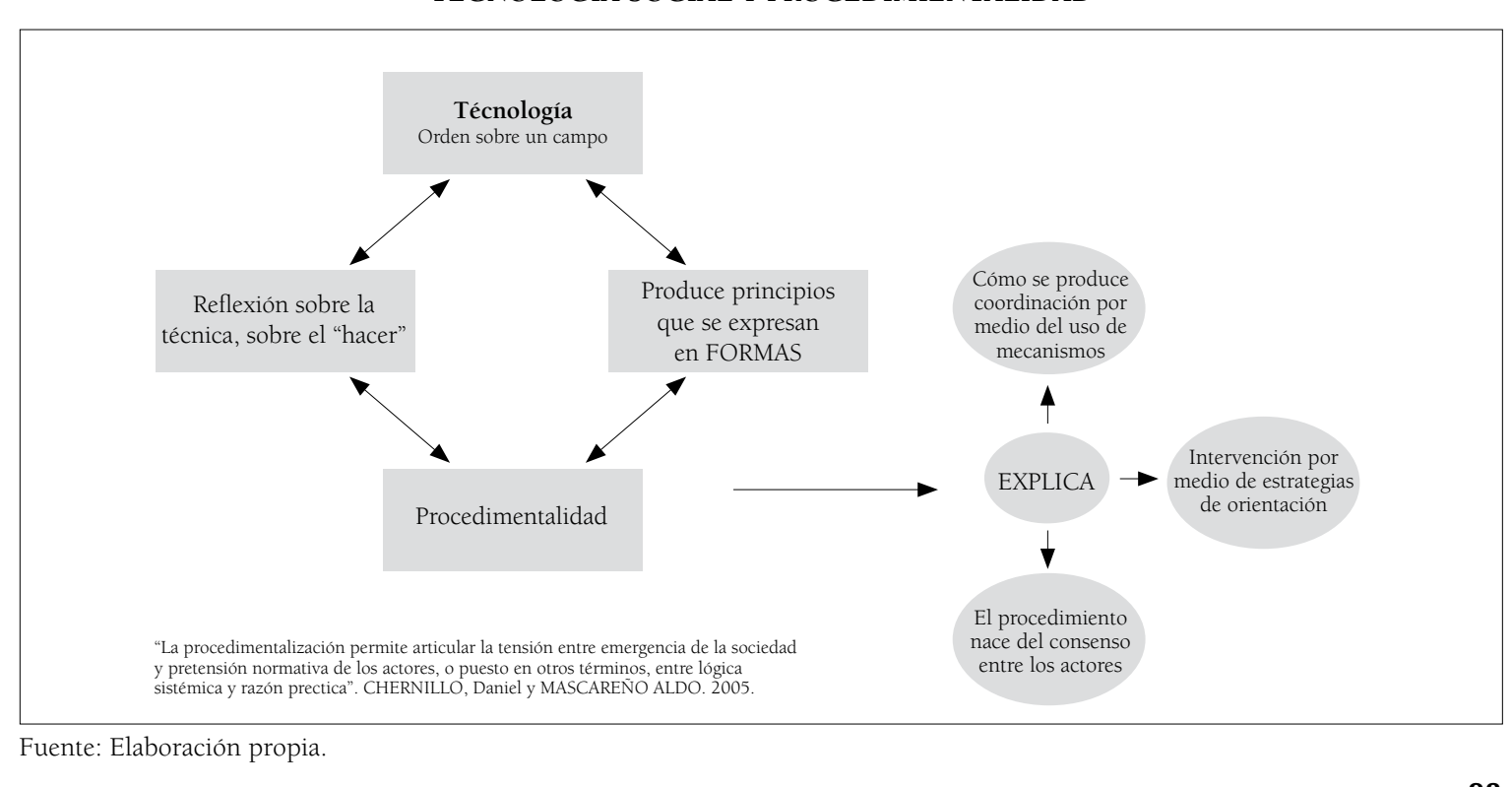

La característica esencial de la intervención social producida es que puede ser estandarizada y ajustada permanentemente a través de una relación entre

Estas tecnologías pueden ser introducidas por la institución, cuando por ejemplo, se diseñan e implementan programas sofisticados y que cuentan con altos márgenes de decisión de los operadores, que cuentan con apoyos interministeriales o mesas de trabajo.

Sin perjuicio de lo anterior, las tecnologías pueden ser introducidas también por el interventor. Sucede esto cuando, por ejemplo, el interventor cuenta con competencias técnicas que superan los márgenes de acción y decisión que contempla el programa, ampliando el conjunto de posibilidades de acción por medio de la aplicación subterránea de tecnología.

\section{Procedimentalidad}

Al hablar de intervención social se piensa habitualmente en la existencia de un sujeto capaz de ser influido o "cambiado" para lograr metas específicas, con o sin la aquiescencia del mismo sujeto. Cuando nos adentramos con más atención en dirección a concepto de procedimentalidad nos vemos obligados a dejar esta noción de sujeto de lado, modificar las lentes con las que habitualmente entendemos la intervención. vas lentes, la dimensión teórica anclada en esta lógica que supone ciertas limitaciones acerca de la manera habitual de entender la intervención y el sujeto. operador y las herramientas trasferidas.

Ello nos invita también a considerar, con estas nue- 
La primera de las imposibilidades respecto del sujeto de intervención dice relación con lo que podríamos llamar "déficits escópicos" (en el modo de ver), es decir, el despliegue de maneras subcomplejas de comprender la intervención social. Este principalmente en acciones dirigidas unidireccionalmente a cambiar "percepciones" a través del cambio de conciencia de las personas. Lo que se sostiene, desde una visión procedimental, es que resulta imposible penetrar en las conciencias de las personas para inocular cambios en sus formas de actuar y pensar. No es la voluntad del sujeto ni mucho menos su conciencia lo que se encuentra en centro de la atención, sino los mecanismos de coordinación dinación entre alter y ego -y no entre un sujeto y un objeto en el sentido cartesiano- , sino coordinación en un sentido comunicativo.

Otro de los factores que incide en estos déficits es el que dice relación con la posibilidad de entender la intervención estructurada a partir de lógicas de pensamiento. Esto quiere decir que nuestro acceso lo social se encuentra mediado por las formas que usamos para interpretar lo social. En este sentido la intervención social, al decir de Teresa Matus, corresponde a una forma de ver que se traduce en un hacer particular.

Como es posible dimensionar, no existe una vía única y garantizada de acceso a lo social ni tampoco un interpretación correcta o paradigmática, sin embargo, terpretación que resultan identificables cuando las asimilamos a las lógicas a partir de las cuales se interpreta lo social. En este sentido, y lo que se sostiene en síntesis es: la reflexión sobre lo social es una forma de pensar filosófico y el preguntar por lo filosófico, es una pregunta social, leída en un contexto.

Una de las entradas posibles para describir una intervención social procedimental es comprender lo procedimental en su dimensión histórica, es decir como un tipo de racionalidad que emerge con modernidad y se expresa consecuentemente en las ciencias jurídicas y políticas que explican cómo se produce coordinación en la sociedad por medio del uso de mecanismos (jurídicos y políticos) y estrategias de orientación $\mathrm{O}$ como nos señalan Chernilo y Mascareño (2005):

La procedimentalización permite articular ensión entre emergencia de la sociedad y pretersión normativa de los actores, o puesto en otros

Estos siete puntos han sido analizados y discutidos en las sesiones del núcleo de estudios en procedimentalidad, seminario convocado el área de metodología e innovación de la Fundación Proyecto Propio y la Escuela de Postgrado de Trabajo Social de la Pontificia Universidad Católica de Chile, a cargo de Teresa Matus.

términos, entre lógica sistémica y razón práctica. Es el mecanismo mediante el cual la diferencia encuentra un punto de anclaje lo suficientemente abstracto como para dar espacio efectivo a lo particular, lo suficientemente concreto como para ser empleado en distintos contextos y lo suficientemente neutro como para permitir la formación de criterios de convivencia universalmente aceptables. Es la única estrategia que no sucumbe cuando, por la propia emergencia de la sociedad, ella se orienta en un sentido distinto al acordado o definido procedimentalmente, pues el procedimiento es neutralidad suprimida y a la vez conservada en el acuerdo, pero no es el acuerdo mismo. (Pp. 17-45).

En este sentido, el procedimiento nace del consenso entre los actores: "pues el procedimiento es neutralidad suprimida y a la vez conservada en el acuerdo, pero no es el acuerdo mismo" (p.17)

En ese sentido, pensar en una intervención social procedimental comporta al menos siete claves o requisitos para esta ${ }^{3}$

\section{Introduce variedad desde la intervención social} (oferta) puesto que al negar lo existente como lo único posible, obliga a no pensar solamente desde el mismo y único lugar, sino que sorprende, innova, haciendo sinergia con otras dimensiones de la intervención y los recursos de otros actores incumbentes.

ii. Involucra al otro, puesto que se trata de elecciones realizadas por este otro (en tanto usuario, cliente, beneficiario, audiencia, público, etc.) y al resultar de un proceso de autoobservación desde él, lo procedimental se encuentra sujeto al cálculo de rentabilidad. La imagen de la variedad introducida en la oferta (en los puntos i y ii) viene a invertir la relación entre complejidad de la demanda versus calidad de la oferta: i) cuanto más aumenta la demanda de un servicio, la calidad es inferior y ii) cuanto más aumenta la oferta (en variedad), la demanda es más específica. En este sentido una oferta limitada se torna mucho más acuciosa, en síntesis, se apunta a "customizar" la oferta del servicio. Este giro hacia la calidad permite demostrar que "hacerlo así" es eficiente, es mejor. En este sentido la procedimentalidad implica un estudio sistemático de la oferta. Como en un equipo de alto rendimiento cuyos entrenadores viabilizan las mejores rendimiento del equipo iii. Implica comunicación con el otro, puesto que se trata de una oferta, el canal y los mecanismos que permitan llegar a este están inscritos en la comunicación. En este proceso uno de los actores participantes puede, en cualquier momento, continuar y rechazar la comunicación, sin embargo, este proceso se establece por medio de contenidos que "tengan sentido" para el otro.

iv. Comporta una pedagogía, ya que requiere considerar la adhesión reflexiva del sujeto a la oferta propuesta por la intervención. Y es que si es requisito el pensar contingentemente la intervención social en base a decisiones que hacen los actores, la procedimentalidad considera mecanismos de trasferencia capaces de incluir las distinciones necesarias (en el lenguaje del otro) para que los usuarios hagan la experiencia de la variedad y a la vez escalonen aprendizajes. En otras palabras, se espera que los usuarios dan a usar la intervención. En esta reflexividad radica la especificidad de una intervención social en tanto oferta customizada y con una performance eficiente. La "customización" implica que los oferentes son capaces igualmente de asentir reflexivamente respecto a aquellos elementos que harán más probable la elección de la oferta (intervención social) por parte del cliente. Hablar de "customizació" deste una logica de de "customizalon" desce una ligica de intervención social procedimental dice relación con reconocer y hacerse cargo de los diferentes Otros, otorgando "ofertas" diferenciadas que son capaces de superar la presunción de usuarios como

CUADRO No1

UMBRALES PARA OFERTAS DE SERVICIOS SOCIALES PROCEDIMIENTALES

\begin{tabular}{|l|l|}
\hline Propiedades & Descripción \\
\hline Contingente & $\begin{array}{l}\text { Consideran que la oferta puede ser aceptada o rechazada por los usuarios, por lo tanto, cuentan con } \\
\text { mecanismos que probabilicen su aceptación. }\end{array}$ \\
\hline $\begin{array}{l}\text { Actualización } \\
\text { (up grade) }\end{array}$ & $\begin{array}{l}\text { La oferta considera su propia obsolescencia, incluyendo actualizaciones, es decir, se proyectan como } \\
\text { ofertas con versiones tipo 1.0, 2.0 etc. }\end{array}$ \\
\hline Adaptación & $\begin{array}{l}\text { La oferta considera la necesidad de modelarse respecto a los requerimientos de sus usuarios, que no son } \\
\text { definitivos ni estáticos. }\end{array}$ \\
\hline Lenguaje & La oferta tiene como requisito el ser comprendidas directamente por los usuarios, sin intermediarios. \\
\hline Transferencia & $\begin{array}{l}\text { Las ofertas presentan instrucciones de uso que mejoren el rendimiento del usuario con la oferta, la idea es } \\
\text { siempre facilitar su experiencia con la oferta. }\end{array}$ \\
\hline Despliegue & La oferta involucra al usuario como gestor y no como receptor pasivo de ella. \\
\hline Autoevaluación & $\begin{array}{l}\text { La oferta se proyecta bajo lógicas de automensuración (el la lógica de score) para que el usuario sepa hasta } \\
\text { dónde puede avanzar aún. }\end{array}$ \\
\hline Normatividad & La oferta no impone contenidos o temas a tratar, sin embargo, no excluye ningún tema a priori. \\
\hline Accountability & La oferta comporta sistemas de rendición de cuentas. \\
\hline
\end{tabular}

Fuente: Elaboración propia un corpus homogéneo del que se conocen, a priori, sus requerimientos (necesidades, aspiraciones, demandas, reivindicaciones, etc.)

v. La intervención social (oferta) se brinda en un cruce con el otro, ya que el carácter intermediado cedimental comporta un campo de comunicación y, sobre todo, de coordinación. Al evitar lo prescriptivo (tú debes) el cruce con el cliente responde a una lógica idiomática (comunicar con las distinciodispuestos en la intervención social como oferta.

vi. Es potencial puesto que se propone contar con "lo cial entendida como oferta (chance) del programa. En este sentido no basta con prometer movilidad sino que es necesario transferirla como posibilidad, actualizarla, enseñar cómo se "comienza" a caminar hacia ella. Tomar esto en cuenta quiere decir que el éxito es procesamiento de la frustración por cuanto "celebrar" la "pérdida" quiere decir celebrar que vamos camino hacia lo distinto

vii.Es crítica, el giro ético responde a la posibilidad cierta de que todo lo anterior se dé: en este sentido, la procedimentalidad es crítica, puesto que permite comparar, identificar las habilidades que se busca y potenciarlas. Esto quiere decir que el actor se involucra en los procedimientos.

traducida en procedimientos desde esta perspectiva, se presenta como "productoservicio" con características capaces de responder a:

En síntesis, el ángulo procedimental nos permite obde la producción de variedad que introduce lo prones del Otro) y a los mecanismos de coordinación que aún no existe". Por ejemplo, la movilidad so- 
servar la intervención social (en tanto oferta) para mejorarla, permite captar lo que los actores involucrados buscan y ponerlo logicamente en contacto con el Otro (usuario). Lo procedimental busca producir experiencias del mundo de una forma Otra y conexpendo los intereses de interventor e intervenido. Interroga acerca de las formas de hacer, produciendo variedad, comparación. En este sentido lo ético está puesto como una oferta trasparente que no promete lo que no cumple. Como campo tecnológico de reflexión centra su atención en el estudio de la oferta.

\section{A modo de conclusión}

En síntesis, podemos señalar que las lógicas esencialistas (ancladas en lo que denominamos siguiendo a Adorno, pensamiento identitario) y las lógicas de intervención centradas en tecnologías sociales conviven en una relación de hibridaje y, a la vez, son introducidas por las lógicas subyacentes en las formas de ver que poseen las organizaciones que implementan programas y/o los operadores (profesionales) que en estas se desempeñan.

La principal consecuencia que se extrae de esta situación, está dada por superar la visión de las intervenciones como "posibilidades únicas y suficientes para personas que no tienen otra opción". Este último ejemplo grafica la imposibilidad (para quienes así lo ven) de introducir actualizaciones (up dos esencializadamente.

Podemos señalar así, que:

- Pensar la intervención social desde un principio procedimental supone lógicas de intervención social post convencionales, esto es, ancladas en una visión postmetafísica (Habermas) del conocimiento, capaces de dotar a programas fijos anclados en formas unidimensionales de ver a anclados en formas unidimensionales de ver
los sujetos, de cualidades dinámicas, móviles, los sujetos, de cualidades dinámicas, móviles, cedimentalización de las intervenciones sociales.

Si la intervención social fuese leída como una lucha, sería una como la del David contra Goliat es que el objeto propio de quienes se afanan en social es el capitalismo y sus consecuencias hum social es el capitalismo y sus consecuencias humanas (Marroquin, 2011). Lo primero que concurre la intervención, entonces, es la adversidad. Esta excesiva negatividad demanda una alta especificidad. En otras palabras, escoger las armas y las estrategias con mucho cuidado antes de iniciar el combate.

¿Cuáles son estas herramientas? En mi opinión, las lógicas con las que se articulan las acciones que conforman las intervenciones sociales y su campo, las regiones del conocimiento.
Estos "combates regionales" se desenvuelven en movimiento, es decir, la relación entre las cosas y las lógicas no se ha de buscar en un horizonte en que no pueden ser aprehendidas de una vez y para siempre sino donde parecen responder a lo que Hegel de"En este sentido el camino de las lógicas de intervención es mortal: no es posible innovar para producir estructuras, sino que para aprender de las cosas, moviéndose junto a ellas y con ellas.

\section{Referencias}

ACEVEDO, J. (2006). Meditación acerca de nuestra época: una era tecnica. En E. Sabrovsky, La tecnica en Heidegger. Santiago de Chile: Universidad Diego Portales.

ADORNO, T., y HORKHEIMER, M. (1987). Dialéctica del iluminismo. Buenos Aires: Sudamericana.

BOURDIEU, P. (1995). Razones prácticas. Barcelona: Anagrama.

CARVAJAL, G. (2010). Observaciones historiográficas sobre el diseño de una teoría general de la tecnolog : Aristóteles Beckmann Marx y Kuhn Revista Observaciones Filosóficas. Volumen 11 Issue 1 Disponible en: http:// www observacionesfilosoficas net/observacioneshistoriograficos.htm

CHERNILO, D., y MASCAREÑO, A. (2005). Universalismo, particularismo y sociedad. Obstáculos y perspectivas de a sociología en América Latina. En Persona y Sociedad, XXIX (3), 17-45. Universidad Alberto Hurtado. Santiago. HABERMAS, J. (1990). Pensamiento postmetafisico. Madrid: Taurus.

HALL, R. (2008). Evidence-based practice as social work tecnology. Irish Journal of Social Strudies, 8 (1), 21-29. LECHNER, N. (2007). Tres formas de coordinación social. En Obras escogidas II. Santiago de Chile: LOM.

MARROQUín, A. (2011). Trabajo social como oficio imposible. Normalización, capitalismo y crítica. Revista de Trabajo Social, 80. Santiago de Chile.

MASCAREÑO, A. (2011). Sociología de la intervención: orientación sistémica contextual. Revista MAD (25). Santiago de Chile.

MATUS, T. (2000). Propuestas contemporáneas en trabajo social. Hacia una intervención polifónica. Buenos Aires: Espacio.

MATUS, T. (1995). Experiencia y pobreza en el trabajo social: una lectura frankfurteana. Revista O Social em Questao, XIII (24)

THYEGESEN, N. (2011). An introduction to understanding technology as illusions. New York: Pelgrave.

\section{Trabajo social y relatos de vida. Reflexiones para una intervención social en dinámicas familiares abusivas}

Social work and life stories: Reflections for a social intervention in abusive family dynamics

Patricia Carrasco Urrutia

Trabajadora social, magister en Ciencias Sociales de la Universidad de La Frontera, Temuco, Chile. Doctora en Ciencias Sociales, Universidad Iberoamericana, México D.F. México. Trabajadora Social del Centro Atención Integral a Victimas de Delitos Violentos, Coyhaique,XI Región,CChile.Email: paticau@hotmail.com

\section{Resumen}

Los relatos de vida pueden constituir una herramienta para el abordaje de casos o la intervenció profesional con familias. A continuación se presentan algunos aspectos de la sistematización de una experiencia de trabajo desarrollada con madres de niños-niñas victimas de un delito sexua intrafamiliar. Se trata de una experiencia profesional que busca traspasar los ejes de la producción discursiva explicita de las madres o figura adulta protectora que acompaña al niño o niña, en el proceso terapéutico reparatorio; $y$ busca acceder a las construcciones significantes que organizan la vida familiar de la victima y su madre, de modo que, a través de la formulación de relatos de zar los sistemas vinculares del niño-niña victima y su figura adulta protectora.

Palabras claves. Trabajo social, intervención social, relatos de vida, adulto protector, resignificar experiencias abusivas.

Abstract

Life stories can be a useful tool for professional intervention within families for social works. Thi paper presents the systematization of the work experience with mothers of children who wer victims of intrafamilial sexual abuse. It tries to go further of the explicit discursive production of mothers or of the protector adults that accompanies the abused child during the therapeutic reparatory process. Instead, this paper attempts to access the meaningful constructions that organize the everyday life of victims and their mothers through life stories. These allow to socia workers co-construct and redefine the abusive experiences and reorganize the emotional links between the victims and the protective adults

Key words. Social work, social intervention, life stories, protector adult, mind change abusive experiences.

\section{Introducción}

Los relatos de vida pueden constituir una herramienta para la intervención social con madres de niños, niñas y adolescentes víctimas de delitos sexuales de carácter intrafamiliar ${ }^{1}$. Los profesionales que participan de equipos o duplas psicosociales para el abordaje requipos o duplas psicosociales sexuales, disponen de amplia literatura para el trabajo con víctimas directas: caracterizaciones, tipos de víctimas, sintomatologías, efectos a corto y largo plazo, entre otros, y una formación profesional terapéutica; no obstante, el trabajo con la red familiar generalmente de cargo del trabajador social, dispone de menos herramientas metodológicas y de literatura especializada para dicha intervención.

En tal sentido, los relatos de vida, ampliamente usados en sociología, pueden ofrecernos una posibilida para el abordaje profesional. Bertaux (2005) sostien 